\title{
Outage Probability of Alamouti based Cooperative Communications with Multiple Relay Nodes using Network Coding
}

\author{
Gordhan Das Menghwar ${ }^{1}$ and Christoph F. Mecklenbräuker ${ }^{2}$ \\ ${ }^{1}$ Information Technology Centre \\ Sindh Agriculture University, Tandojam, Pakistan \\ Telephone: +92-22-2766338 \\ Fax: +92-22-2765300 \\ Email: gordhan_das@hotmail.com \\ ${ }^{2}$ Institute of Communications and Radio Frequency Engineering \\ Vienna University of Technology, Vienna, Austria \\ Telephone: +43-1-58801-38996 \\ Fax: +43-1-58801-38999 \\ Email: cfm@nt.tuwien.ac.at
}

\begin{abstract}
In this paper, we extend our proposed scheme of cooperative communications based on Alamouti scheme which was aided by a single relay node to forward network coded bits to the destination. In this work, instead of a single relay node, we consider multiple relays forwarding network coded bits, of the information received from the cooperating users, to the destination. With analytical results on the outage probability, we show that the cooperative diversity gain of the proposed scheme increases linearly with $m$, the number of relay nodes assisting cooperating users by forwarding network coded bits of their information to the destination.
\end{abstract}

\section{INTRODUCTION}

Due to the promising gains offered by cooperative communications and the relay channel, the standard bodies for future generation wireless communications, like LTE-Advanced [1] and IEEE802.16j [2][3], have shown great interest in cooperative transmission and in the use of a fixed relay node [4] in the network. With cooperative transmission between the base stations, it is planned to reduce the interference effects especially for the edge users [5][6]. In this way, the edge users are intended to not only have higher throughput but increased reliability as well. In the same way, the use of a relay node is also proposed to offer higher data rates and to cover the coverage holes for the users at the cell boundary [3].

On the other hand, in user cooperation or cooperative communications setup, users in common vicinity share their resources to achieve the same goal like the diversity gain and the improved throughput. Cooperative communications is a way of transmission where the users help each other for reliable recovery of their messages at the destination [7][8][9].

Based on the fact that the future generation wireless systems are expected to have fixed relay node installed in the network, we proposed a scheme which combines the concept of cooperative communications, which does not require any dedicated terminal, and the use of a fixed relay node in the network [10][11][12].

To implement that scheme, we used two cooperating users using space-time coding based Alamouti scheme and a fixed relay node forwarding network coded bits to the destination. Network coding is an idea based on mixing the data at the intermediate nodes when travelling from source to the destination [13]. In a wireless network, like ad hoc or cellular systems, the available free users can also be considered as fixed relay nodes.

In particular, in [12], we investigated the proposed scheme using outage probability as a performance measure. It was shown that with this combination of cooperative communications and fixed relay node, we can outperform cooperation using Alamouti scheme only. While our previous work was based on the availability of a single relay node, in this paper, we extend the number of available relay nodes assisting the cooperating users. As a performance analysis for slow fading channel, we derive the resulting outage probability of the proposed scheme. The results indicate that there is a linear increase in the diversity order of the scheme with every increase of the relay node.

The rest of the paper is structured as follows. Section II describes our system model. Section III gives the performance analysis of the scheme. In Section III-A, we give the mutual information and in Section III-B the resulting outage probability of the proposed scheme is derived. The results are presented and discussed in Section IV. Finally, Section V draws the conclusion of our paper.

\section{System Model}

We consider a cellular network with two cooperating users $u_{1}$ and $u_{2}$, single base station, and multiple relays or free users in their vicinity, as shown in Fig. 1. Since 


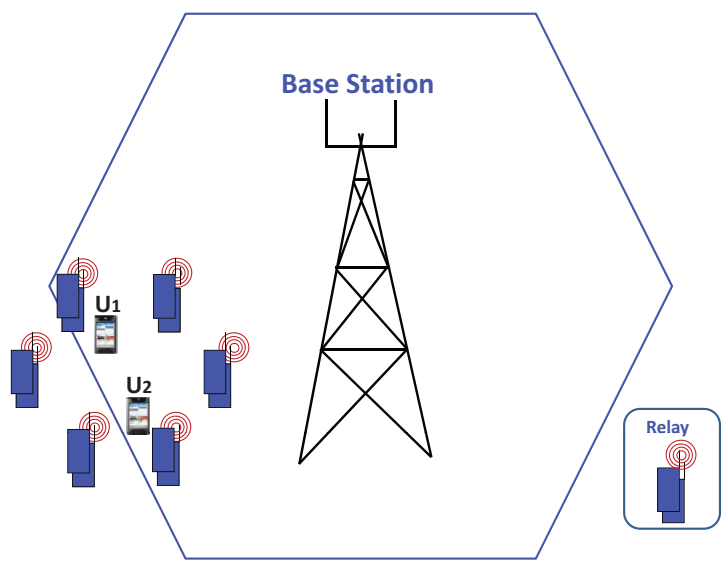

Fig. 1. Cooperative communications setup exploiting the availability multiple dedicated relay nodes

our scheme builds upon cooperative communications using Alamouti scheme [14], first we briefly go through this scheme and then discuss our investigated strategy.

Fig. 2 shows cooperative communications setup showing two users using space-time coding based Alamouti scheme. Transmission of the message is assumed to take place in two phases. In the first phase, each of the user, in turn, transmits

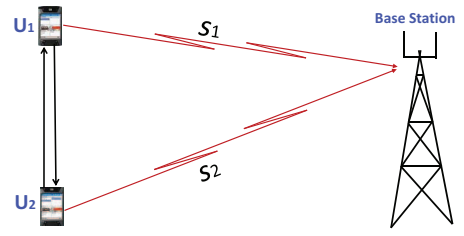

(a) First Phase

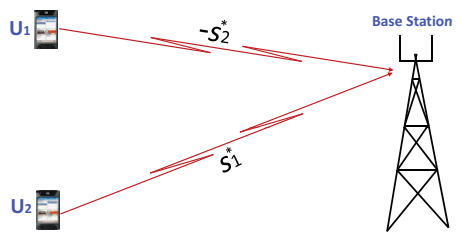

(b) Second Phase

Fig. 2. Space-time Cooperative communications using Alamouti scheme

its own message $s_{1}$ and $s_{2}$, respectively, to the destination, as depicted in Fig. 2(a). This message, along with the intended destination which is base station in our case, is also received at each other's receivers. In the next phase, both of the users, act as a relay node for each other. And following Alamouti scheme, send $-s_{2}^{*}$ and $s_{1}^{*}$, respectively, to the destination, as illustrated in Fig. 2(b). The sign of $*$ represents the complex conjugate operation.

As a result, the destination receives two copies of the same message transmitted from the transmitting users. These two copies of the message, based on the channel condition, are received at the destination from two independently fading paths and offer diversity order of two.
In our scheme, in addition to the cooperating users and the destination, we also introduce multiple relay nodes in the network, as shown in Fig. 3.

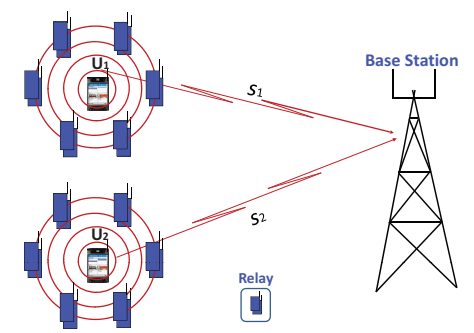

(a) First Phase

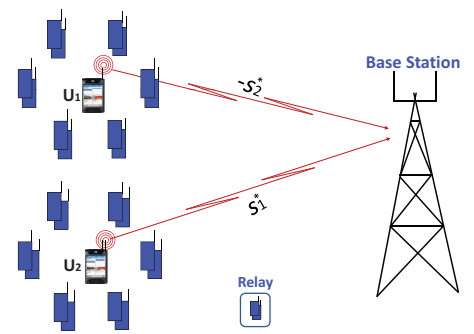

(b) Second Phase

Fig. 3. Space-Time Cooperative communications using Alamouti scheme with network coding

Again the transmission of the message is accomplished in two phases. During first phase, the cooperating users transmit $s_{1}$ and $s_{2}$, respectively to the destination. This time due to the presence of multiple relays in the vicinity of the transmitting users, the messages are also received at these relay nodes. This is shown in Fig. 3(a). Later in the next phase, the cooperating users transmit $-s_{2}^{*}$ and $s_{1}^{*}$, respectively to the destination. In the same phase, all of the relay nodes decode the messages received from the cooperating users and forward network coded bits of those decoded messages to the destination, as illustrated in Fig. 3(b). To avoid the interference, the mobile station users and all of the relay nodes jointly are assigned orthogonal channels. Although this orthogonality can equally be achieved by using different time, frequency or code, as is done in today's cellular networks, we focus here only on time based orthogonality.

Table I shows the operational details of our proposed scheme. $r_{j}$ represents the $j^{\text {th }}$ relay node, where $j=$ $1,2, \ldots, m$. For instance, the first row of Table I indicates that

TABLE I

THE OPERATIONAL DETAILS OF THE SCHEME

\begin{tabular}{|c|c|c|c|}
\hline Time Slot & Transmitting Node & Transmitted Message & Receiving Node \\
\hline \hline 1 & $u_{1}$ & $s_{1}$ & $u_{2}, \mathrm{BS}, r_{1}, \ldots r_{m}$ \\
2 & $u_{2}$ & $s_{2}$ & $u_{1}, \mathrm{BS}, r_{1}, \ldots r_{m}$ \\
3 & $u_{1}$ & $-s_{2}^{*}$ & $\mathrm{BS}$ \\
4 & $u_{2}$ & $s_{1}^{*}$ & $\mathrm{BS}$ \\
5 & $r_{1}, \ldots r_{m}$ & $s_{1} \oplus s_{2}$ & $\mathrm{BS}$ \\
\hline
\end{tabular}

during first time slot user $u_{1}$ transmits $s_{1}$ and user $u_{2}$, base 
station (BS), and all of the relay nodes receive this message. Likewise in the next time slot, user $u_{2}$ transmits $s_{2}$; this time user $u_{1}, \mathrm{BS}$, and the relay nodes act as receiving nodes for this message. Finally, in the fifth time slot, all of the relay nodes transmit network coded bits of the information received from the cooperating users to the destination. Symbol of $\oplus$ is used to show Ex-OR operation to implement the idea of network coding.

We consider slow fading channel and the fading coefficients are modeled as zero mean complex Gaussian random variables with zero mean and $\sigma^{2}$ variance. In addition to that, additive white Gaussian noise (AWGN) with zero mean and unit variance is assumed at each receiver, i.e., at the receivers of the cooperating mobile station users and at the destination. Both of the cooperating users and all of the relay nodes comply with a power restriction of $P_{i}$. Furthermore, perfect channel state information is assumed at the receiving end, but not at the transmitters.

Signal-to-noise ratio (SNR) is defined as

$$
\gamma\left|h_{i, j}\right|^{2}
$$

where

$$
\gamma=\frac{P_{i}}{N_{o}}
$$

\section{Performance Analysis}

In this section, we analytically evaluate the performance of our proposed scheme, i.e., cooperative communications using Alamouti scheme with network coding when multiple numbers of relay nodes are present in the network. As mentioned before, these relay nodes forward network coded bits, of the messages received from the cooperating users, to the destination.

For performance evaluation purpose, first we evaluate the analytical expression for the mutual information between the cooperating user $u_{i}$ and the destination. Later on the basis of mutual information, we derive the outage probability for the investigated scheme.

\section{A. Mutual Information}

For simplicity, we assume that the channel between the cooperating users and from the cooperating users to the relay nodes are error free.

Based on this assumption, the mutual information, for the scheme with two cooperating users using space-time codes and multiple relays forwarding network coded bits to the destination, can be written as ${ }^{1}$

$$
I=\frac{2}{5} \log \left(1+\frac{\gamma}{2} \sum_{i=1}^{2}\left|h_{i, 0}\right|^{2}\right)+\frac{2}{5} \log \left(1+\gamma \sum_{i=1}^{m}\left|h_{r, 0}\right|^{2}\right) .
$$

$h_{i, 0}$ and $h_{r, 0}$ shows the channel gain from $i^{t h}$ cooperating user and $r^{t h}$ relay channel, respectively, to the destination. Here, the first of the two constituent terms is due to the Alamouti scheme [15], and the second term is due to network coded bits, forwarded from the relay nodes to the destination [16]. The factor $\frac{2}{5}$, in front of the mutual information, indicates that five time slots are used by two cooperating users to transmit their messages to the destination. Here four time slots are used when cooperating users use space-time codes and additional one time slot is used by the $m$ relay nodes which forward network coded bits to the destination. As described in Table I, all of the relay nodes are considered to transmit simultaneously.

\section{B. Outage Probability}

As we know the maximum transfer rate is dependent on the mutual information between input and output [17]. Mutual information expression (2) indicates the fact that the achieved rate is dependent on the channel gain, which is considered to follow Rayleigh distribution and is a random variable. Following [18], we define the outage probability to be the probability when the channel cannot meet certain rate requirement $R$, demanded by some particular application, like video or some multimedia application.

Mathematically it can be written as [19][20]

$$
P[R]=P[I<R]
$$

With the help of (2) and (3), we derive the outage probability of the proposed scheme as follows:

$$
\begin{aligned}
P[R] & =P[I<R] \\
& =P\left[\frac{2}{5} \log \left(1+\frac{\gamma}{2} \sum_{i=1}^{2}\left|h_{i, 0}\right|^{2}\right)+\frac{2}{5} \log \left(1+\frac{\gamma}{m} \sum_{i=1}^{m}\right.\right. \\
& =P\left[\frac{2}{5} \log \left(1+\left.\frac{\gamma}{2} \sum_{i=1}^{2}\left|h_{i, 0}\right|^{2}\right|^{2}\right)\left(1+\frac{\gamma}{m} \sum_{i=1}^{m}\left|h_{r, 0}\right|^{2}\right)<R\right] \\
& =P\left[1+\frac{\gamma}{m} \sum_{i=1}^{m}\left|h_{r, 0}\right|^{2}+\frac{\gamma}{2} \sum_{i=1}^{2}\left|h_{i, 0}\right|^{2}+\frac{\gamma^{2}}{2 m} \sum_{i=1}^{2}\left|h_{i, 0}\right|^{2}\right. \\
& =P\left[\frac{1}{m} \sum_{i=1}^{m}\left|h_{r, 0}\right|^{2}+\frac{1}{2} \sum_{i=1}^{2}\left|h_{i, 0}\right|^{2}+\frac{\gamma}{2 m} \sum_{i=1}^{2}\left|h_{r, 0}\right|^{2}<2^{\frac{5}{2} R}\right] \\
& \left.\left|h_{r, 0}\right|^{2}<\frac{2^{\frac{5}{2} R}-1}{\gamma}\right]
\end{aligned}
$$

Let $\sum_{i=1}^{m}\left|h_{r, 0}\right|^{2}=x, \sum_{i=1}^{2}\left|h_{i, 0}\right|^{2}=y$, and $\frac{2^{\frac{5}{2} R}-1}{\gamma}=\epsilon$. 
Then

$$
\begin{aligned}
P[R] & =P\left[\frac{1}{m} x+\frac{1}{2} y+\frac{1}{2 m} \gamma x y<\epsilon\right] \\
& =P\left[y\left(\frac{1}{2}+\frac{1}{2 m} \gamma x\right)<\epsilon-x\right] \\
& =P\left[y<\frac{\epsilon-\frac{1}{m} x}{\frac{1}{2}+\frac{1}{2} \gamma \frac{1}{m} x}\right] \\
& =\int_{0}^{\epsilon m} P\left[y<\frac{\epsilon-\frac{1}{m} x}{\frac{1}{2}+\frac{1}{2} \gamma \frac{1}{m} x} \mid x\right] P_{X}(x) d x \\
& =\int_{0}^{\epsilon m}[1-\exp (g h)] P_{X}(x) d x
\end{aligned}
$$

where

$$
g=\left(-\lambda \frac{\epsilon-\frac{1}{m} x}{\frac{1}{2}+\frac{1}{2} \gamma \frac{1}{m} x}\right)
$$

and

$$
h=\left(1+\lambda \frac{\epsilon-x}{\frac{1}{2}+\frac{1}{2} \gamma \frac{1}{m} x}\right) .
$$

The second last line of the equation (4) is due to the law of total probability theorem; and the last equation is obtained by using the cumulative distribution function (cdf) for the sum of two exponential random variables, represented by $y$. A general form of cdf for the sum of $k$ exponential random variables is given by [21]

$$
F(x)=1-\exp (-\lambda x) \sum_{k=0}^{k-1} \frac{(\lambda x)^{k}}{k !} .
$$

$P_{X}(x)$ represents the probability distribution function (pdf) for the sum of $m$ independent exponential random variables and is given by [21]

$$
P_{X}(x)=\left\{\begin{array}{cc}
\frac{\lambda}{(m-1) !}(\lambda x)^{m-1} \exp (-\lambda x) & \text { for } x>0 \\
0 & \text { for } x \leq 0
\end{array}\right.
$$

where $\lambda>0$ represents the parameter of the exponential distribution.

\section{RESUlts AND Discussion}

In Figure 4, we show the outage probability results, based on the expression (4), evaluated at different values of $\mathrm{SNR}^{2}$.

Without loss of generality, $\lambda$ is taken to be 1 , and the required spectral efficiency $R$, is fixed at $1 \mathrm{~b} / \mathrm{s} / \mathrm{Hz}$. As depicted, the relative diversity gains offered by non-cooperative transmission and the cooperative communications using Alamouti scheme are also shown.

The diversity order of a particular scheme can be seen as the slope of the outage probability curve [22]. With this definition, we can see that the non-cooperative communications and cooperative communication using Alamouti scheme offer diversity order of one and two, respectively. On the other hand, the diversity gain offered by cooperative communications using

\footnotetext{
${ }^{2}$ In the figure, the numerals ( $\left.m=1,2,5,7\right)$ show the number of relay nodes used to forward network coded bit to the destination.
}

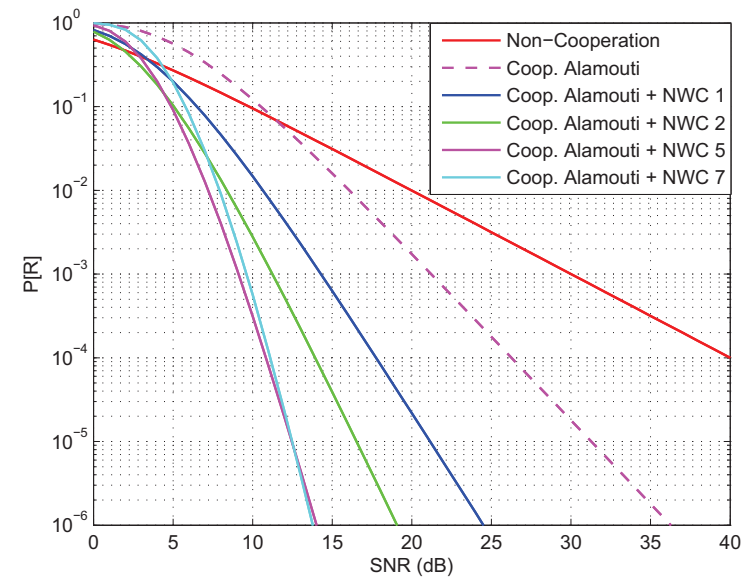

Fig. 4. Outage probability for cooperative communications using Alamouti scheme with network coding aided by $1,2,5$, and 7 relay nodes in comparison with the outage probability for direct transmission and cooperative communication using Alamouti scheme without network coding. The required spectral efficiency $R$ is set to $1 \mathrm{~b} / \mathrm{s} / \mathrm{Hz}$.

Alamouti scheme with network coding aided by $m$ relay nodes is of the order of $m+2$. In the figure, we have shown the outage probability curves for $m=2,5$, and 7 which happens to be of the order of 4, 7, and 9, respectively. Furthermore, in the Figure 4, it is easy to see, with the help of the slope of outage probability curves, that there is a linear increase in the diversity order with every increase of the relay node.

\section{CONCLUSION}

We extended our work on cooperative communications using Alamouti scheme aided by a single relay to multiple relay nodes. As a performance measure, we used outage probability of the scheme. The derived results indicate that the achieved diversity gain outperforms the diversity gain achieved with cooperative communications using Alamouti scheme only. Furthermore, this gain increases linearly with the number of relay nodes, assisting the cooperating users by sending the network coded bits of their messages to the destination.

\section{REFERENCES}

[1] 3GPP TR 36.814 V1.2.1, "Further advancements for EUTRA: Physical layer aspects," Tech. Spec.n Group Radio Access Network, June 2009.

[2] I. P802.16j/D9, "Draft amendment to IEEE standard for local and metropolitan area network part 16: Air interface for fixed and mobile broadband wireless access systems: Multihop relay specification," Feb 2009.

[3] Y. Yang, H. Hu, J. Xu, and G. Mao, "Relay technologies for WiMAX and LTE-Advanced mobile systems," IEEE Communications Magazine, vol. 47, no. 10, pp. 100-105, October 2009.

[4] T. Cover and A. E. Gamal, "Capacity theorems for the relay channel," IEEE Trans. Inform. Theory, vol. IT-25, no. 5, pp. 572-584, Sep 1979.

[5] S. Parkvall and D. Astely, "The evolution of LTE towards IMTAdvanced," Journal of Communications, vol. 4, no. 3, pp. 146-154, April 2009. 
[6] D. Martn-Sacristn, J. F. Monserrat, J. Cabrejas-Peuelas, D. Calabuig, S. Garrigas, and N. Cardona, "On the way towards fourth-generation mobile: 3GPP LTE and LTE-Advanced," EURASIP Journal on Wireless Communications and Networking, vol. 2009, pp. Article ID 731317, 11 pages, 2009.

[7] A. Sendonaris, E. Erkip, and B. Aazhang, "User cooperation diversityPart I: System description," IEEE Trans. Commun., vol. 51, no. 11, pp. 1927-1938, Nov 2003.

[8] - "User cooperation diversity-Part II: Implementation aspects and performance analysis," IEEE Trans. Commun., vol. 51, no. 11, pp. 19391948, Nov 2003.

[9] T. E. Hunter and A. Nosratinia, "Cooperation diversity through coding," Proceedings IEEE International Symposium on Information Theory, $\mathrm{p}$. 220, July 2002.

[10] G. D. Menghwar, B. Krasniqi, A. A. Shah, and C. F. Mecklenbräuker, "Cooperative space-time codes with opportunistic network coding," Proceedings of IEEE Sarnoff Symposium 2009, Princeton, New Jersey, USA, April 2009. [Online]. Available: http://www.http://publik.tuwien. ac.at/files/PubDat 176211.pdf/

[11] G. D. Menghwar, A. A. Shah, and C. F. Mecklenbräuker, "Cooperative space-time codes with opportunistic network coding with increasing numbers of nodes," Proc. IEEE Sixth International Symposium on Wireless Communication Systems 2009 (ISWCS-2009), Sep 2009.

[12] G. D. Menghwar and C. F. Mecklenbräuker, "Outage analysis of cooperative space-time codes with network coding," in International ITG Workshop on Smart Antennas (WSA 2010), Bremen, Germany, Feb. 2010.

[13] R. Ahlswede, C. Ning, S.-Y. R. Li, and R. W. Yeung, "Network information flow," IEEE Trans. Inform. Theory, vol. 46, no. 4, pp. 1204 1216, July 2000.

[14] S. M. Alamouti, "A simple transmit diversity technique for wireless communications," IEEE Journal on Selected Areas in Communications, vol. 16, no. 8, pp. 1451-1458, 1998. [Online]. Available: http: //dx.doi.org/10.1109/49.730453

[15] B. Hassibi and B. M. Hochwald, "High-rate codes that are linear in space and time," IEEE Trans. on Inform. Theory, vol. 48, no. 7, pp. 1804-1824, 2002.

[16] X. Bao and J. L. (Tiffany), "Adaptive network coded cooperation (ancc) for wireless relay networks: matching code-on-graph with network-ongraph," IEEE Trans. on Wireless Communications, vol. 7, no. 2, pp. 574-583, Feb 2008.

[17] T. M. Cover and J. A. Thomas, Elements of information theory. John Wiley \& Sons, Inc., New York, 1991.

[18] L. H. Ozarow, S. Shamai, and A. D. Wyner, "Information theoretic considerations for cellular mobile radio," IEEE Trans. on Vehicular Technology, vol. 43, no. 2, pp. 359-378, May 1994.

[19] J. N. Laneman, G. W. Wornell, , and D. N. C. Tse, "An efficient protocol for realizing cooperative diversity in wireless networks," in proc. IEEE Int. Symp. Information Theory, p. 294, June 2001.

[20] J. N. Laneman, D. N. C. Tse, and G. W. Wornell, "Cooperative diversity in wireless networks: efficient protocols and outage behavior," IEEE Trans. Inform. Theory, vol. 50, no. 12, pp. 3062-3080, Dec 2004.

[21] A. Papoulis, Probability, Random Variables, and Stochastic Processes. Mc-Graw Hill, 1984.

[22] D. Tse and P. Viswanath, "Fundamentals of wireless communication." Cambridge University Press, Jun 2005. 